Winter Meeting - Joint meeting between the Nutrition Society and the Royal Society of Medicine, 11-12 December 2012,

\title{
An assessment of the Atkins diet on skeletal health in contrast to diets rich in alkaline-forming fruits and vegetables
}

\author{
C. Huggett ${ }^{1}$, R. H. T. Gannon ${ }^{2}$, H. Truby ${ }^{1}$, R. Hiscutt ${ }^{1}$, H. Lambert ${ }^{1}$, W. D. Fraser $^{3}$ \\ and S. A. Lanham-New ${ }^{1}$ \\ ${ }^{1}$ Department of nutrition and metabolism, University of Surrey, GU2 $7 \mathrm{XH},{ }^{2}$ Department of health and nutrition, \\ Nestlé research centre, Switzerland and ${ }^{3}$ Faculty of medicine and health sciences, University of East Anglia, NR4 7TJ, UK
}

It has been suggested that the human skeleton plays an important role in acid-base balance ${ }^{(1)}$ and that eating a diet plentiful in alkalineforming foods, notably fruit and vegetables, may provide benefits for bone health ${ }^{(2,3,4)}$. In contrast, a dietary pattern typical of Western societies that is insufficient in fruit and vegetables, but is high in acid-forming protein may present a mild metabolic acidosis and thus deleterious effects to skeletal health ${ }^{(5)}$.

The data used within this project were collected from an intervention study ${ }^{(6)}$, which investigated the effects of subjects following either the Atkins diet, a weight-reduction diet high in fruit and vegetables (HFV) or a control diet: on markers of bone turnover (BTM) over 6 months, in 67 UK overweight men (BMI $27-40 \mathrm{~kg} / \mathrm{m}^{2}$ ). Statistical analyses were performed for differences in diet and acidity (potential renal acid load (PRAL) and net endogenous acid production (NEAP) using the protein: potassium algorithm), in association with BTMs, over time and between groups: free Pyridinoline relative to Creatine (fPYD/Creatine), free Deoxypridinoline relative to Creatine (fDPD/ Creatine), serum C-telopeptides of collagen (CTx) and serum amino-terminal procollagen extension peptide (P1NP) were measured.

Both the Atkins and HFV diet groups experienced significant reductions in weight and a significant increase was seen over time for potassium intake for the HFV diet $(P<0.030)$. Significant increases were observed in the Atkins group for PRAL and for NEAP $(P<0.045$ $\& P<0.043)$, while significant decreases were seen for the HFV diet respectively $(P<0.034 \& P<0.033)$ (see Fig. 1). A significant increase was demonstrated for CTx in both Atkins and HFV $(P<0.012 \& P<0.002)$ and a trend was seen concerning elevated CTx, for subjects with NEAP estimates 1 SD above the mean $(P<0.093)$ (see Fig. 2).

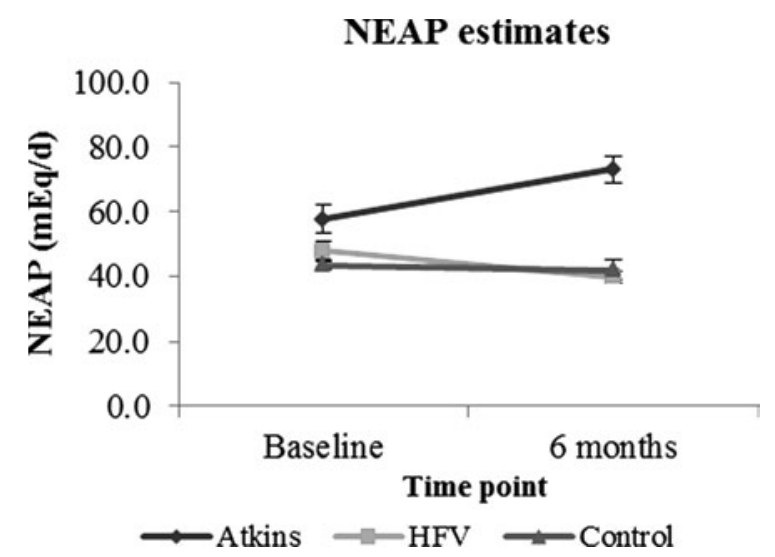

Fig. 1. NEAP estimates (mEq/d) (Paired t-tests).

\section{CTx levels according to NEAP estimates}

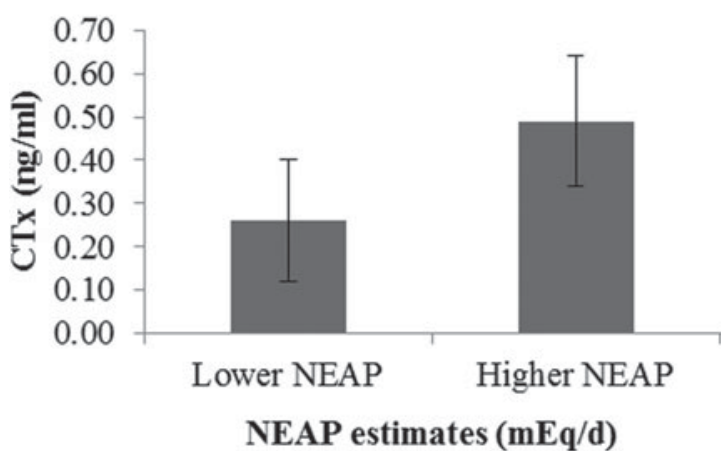

Fig. 2. CTx (ng/ml) according to high or low NEAP (1SD above or below mean estimate).

The results confirmed that the Atkins diet is significantly more acidic than the HFV diet and this appeared to increase bone resorption. Conversely, HFV provides an alkaline diet, rich in potassium but it did not demonstrate reductions in bone resorption. The NEAP trend observed warrants further investigation as numbers were small; indeed future research may be able to establish if HFV offers benefits for bone-associated outcomes and thus osteoporosis prevention.

1. Wachman \& Bernstein (1968) The Lancet. 291, 958-959.

2. Tucker et al. (1999) Am J Clin Nutr. 69, 727-736.

3. New et al. (1997) Am J Clin Nutr. 65, 1831-1839.

4. Chen et al. (2006) Brit J Nutr. 96, 745-751.

5. Remer et al. (2000) Semin Dialysis. 13, 221-226.

6. Gannon et al. (2007) Osteoporosis Int. 18, Suppl 3. 